\title{
Cardiovascular effects of mannitol infusion: a comparison study performed on mouse and human
}

Teemu Myllylä, Mika Kaakinen, Aleksandra Zienkiewicz, Jari Jukkola, Erkki Vihriälä, et al.

Teemu Myllylä, Mika Kaakinen, Aleksandra Zienkiewicz, Jari Jukkola, Erkki Vihriälä, Vesa Korhonen, Outi Kuittinen M.D., Lauri Eklund, Vesa Kiviniemi M. D., "Cardiovascular effects of mannitol infusion: a comparison study performed on mouse and human," Proc. SPIE 10685, Biophotonics: Photonic Solutions for Better Health Care VI, 106854A (17 May 2018); doi: $10.1117 / 12.2307380$ 


\title{
Cardiovascular effects of mannitol infusion - a comparison study performed on mouse and human
}

\author{
Teemu Myllylä*a,b, Mika Kaakinen ${ }^{\mathrm{c}}$, Aleksandra Zienkiewicz ${ }^{\mathrm{b}}$, Jari Jukkola ${ }^{\mathrm{c}}$, \\ Erkki Vihriäläb, Vesa Korhonen ${ }^{\mathrm{a}, \mathrm{d}}$, Outi Kuittinen ${ }^{\mathrm{e}}$, Lauri Eklund ${ }^{\mathrm{c}}$, Vesa Kiviniemi ${ }^{\mathrm{a}, \mathrm{d}}$ \\ ${ }^{a}$ University of Oulu, Research Unit of Medical Imaging, Physics and Technology, Oulu, Finland; \\ ${ }^{b}$ University of Oulu, Optoelectronics and Measurement Techniques Unit, Oulu, Finland; ' ${ }^{\circ}$ niversity \\ of Oulu, Biocenter Oulu, Faculty of Biochemistry and Molecular Medicine, Oulu, Finland.; ${ }^{\mathrm{d}}$ Oulu \\ University Hospital, Department of Diagnostic Radiology, Medical Research Center (MRC), Oulu, \\ Finland; 'University of Oulu, Institute of Clinical Medicine, Oulu, Finland
}

\begin{abstract}
Monitoring blood-brain barrier (BBB) opening is of great interest in terms of brain drug delivery in the treatment of brain lymphoma and maybe in the future in other diseases like dementia. A method involving BBB disruption (BBBD) by mannitol infusion has been developed in University of Portland, USA, and then exploited in Oulu University Hospital in treatment of primary CSN lymphoma. Proper opening of the BBB is crucial for the treatment, yet there are no methods available for its real-time clinical monitoring. Recently, we presented a combined method using direct-current electroencephalography (DC-EEG) and near-infrared spectroscopy (NIRS) for monitoring BBBD in human. Carotid artery mannitol infusion generated a strongly lateralized DC-EEG response and in NIRS a prolonged increase in the oxy/deoxyhemoglobin ratio.
\end{abstract}

This study explores further BBBD, by focusing on monitoring its cardiovascular effects, when measured in human and mouse. For this, we used photoplethysmography (PPG) and opto-electro-mechanical sensors to gather the signals in human and mouse. Mannitol infusion in human causes strong fluctuations in blood pressure, heart rate and PPG signals, and here we discuss how the acquired signals in mouse model compares to human data.

In addition, we present our scale-free monitoring concept that enables monitoring physiological signals similarly when performing experiments in mouse and human neuroimaging setups. By combining microscopic and macroscopic imaging in mouse setup enables us to study correlations between mechanistic cellular data and clinical functional data. Further, this allows us to validate and optimize macroscopic sensing and imaging techniques aimed to be used in human imaging.

Keywords: blood-brain barrier, PPG, opto-electro-mechanical sensors, cardiovascular system, clinical monitoring

\section{INTRODUCTION}

The BBBD [1] by intra-arterial mannitol infusion provides first efficient treatment for otherwise lethal disease in treatment of primary CSN lymphoma (PCSNL) patients [2,3]. Currently, other opening methods, such as, focused ultrasound (FUS) [4] and photodynamic treatment (PDT) [5] are still in preclinical development. Mannitol infusion in PCSNL patients generates a strong DC change in EEG response and a prolonged increase in the oxy/deoxyhemoglobin ratio in the brain cortex [6]. However, it is not yet clear what is the isolated role of BBBD in the observed signals, moreover, is the response of other BBB opening methods similar. In the BBBD PCSNL procedure, also very strong fluctuations in blood pressure (BP), heart rate (HR) and vascular pulsations can be observed, which are assumed to be caused mostly due to mannitol infusion. In order to optimize and improve safety of intra-arterial mannitol infusion based BBBD in clinical use controlling of cardiovascular loading during the BBBD procedure is of great interest.

Although there are studies on effects of mannitol administration on cardiovascular health, they are based only on animal data. For instance, Garcia-Sola et al. showed that infusion of mannitol in goat causes strong elevation of BP: at first, immediate rise in BP, next strong decrease and then recovering, however, to a lower BP value than pre infusion [7].

*teemu.myllyla@oulu.fi; phone +358 294482762

Biophotonics: Photonic Solutions for Better Health Care VI, edited by Jürgen Popp,

Valery V. Tuchin, Francesco Saverio Pavone, Proc. of SPIE Vol. 10685, 106854A

(C) 2018 SPIE · CCC code: $0277-786 \mathrm{X} / 18 / \$ 18 \cdot$ doi: $10.1117 / 12.2307380$

Proc. of SPIE Vol. 10685 106854A-1 
Another paper, by Gumerlock and Neuwelt, evaluates the effect of various anesthetic agents in Sprague-Dawley rats when used with mannitol. Rats were given pentobarbital, ketamine-xylazine, isoflurane, methoxyflurane, or fentanyldroperidol before intracarotid infusion of mannitol. In general, in all of these anesthetic agents, post infusion effects of mannitol were a strong decrease in BP and heart rate, and then their increase near to values pre infusion [8]. This study also suggested that the cardiovascular changes of these specific agents are important in obtaining optimal hyperosmolar BBBD.

Our aim is to develop non-invasive measurement techniques that enables monitoring effects of BBBD similarly from mouse and human. This multimodal setup can be used to optimize and improve safety of BBBD methods for clinical use, moreover, it supports enhancing the BBBD monitoring that is currently in clinical use. We will present our cardiovascular monitoring setup for mouse and human BBBD studies. Further, results show cardiovascular effects of mannitol infusion, measured from both mouse and human. Responses of HR, breathing and vascular pulsation are measured using opto-electro-mechanical sensing techniques. In addition, BP was measured on human patients invasively.

\section{METHODOLOGY}

\subsection{Method of measurement}

In mouse, as presented on left in Figure 1, breathing was monitored by small opto-mechanical accelerometers placed on chest and abdomen. HR was measured both by electrocardiography (ECG) with 3 leads and by accelerometer placed on chest. Vascular pulsation (changes in arterial blood volume) was measured using a NIRS optode placed on head. Distance between the light source and detector was $5 \mathrm{~mm}$. In this paper, we only show vascular pulsation, which can be also measured by a single-wavelength PPG sensor. On the other hand, by using a combination of two or three 3 wavelengths signal quality can be improved when it is disturbed by motion [9], which we also aim to study. However, these results show vascular pulsation gathered using wavelength of $940 \mathrm{~nm}$. Vascular pulsation was measured from mouse head where less motion artefacts were present.

Accelerometers can detect both HR and breathing rate, but is also very sensitive to motion artefacts. Most of the motion artefacts were apparently caused by breathing, which could be seen as a strong motion of abdomen. For this reason, breathing patterns were measured by accelerometer placed on abdomen and then by low pass filtering the signal with a cutoff frequency of $150 \mathrm{~Hz}$. Using similar technique, breathing patterns can be measured also from human as presented for instance in [10]. Furthermore, HR can be measured by accelerometer placed on chest, when high-pass filtering frequencies above the frequency range of breathing.

In human, similar sensor placement for accelerometers and NIRS was used, where NIRS optode on forehead had sourcedetector distance of $3 \mathrm{~cm}$. In addition, ECG, intra-arterial BP and PPG from index finger were recorded using GE DatexOhmeda S/5 Avance setup [6]. Hemodynamic responses of the NIRS signals are presented in [6]. Vascular pulsation, presented here in Figure 2, are gathered by the PPG sensor placed on index finger. During the BBBD procedure, human patients breathing is supported by a ventilator machine thus the constant breathing patterns during anesthesia are here not presented.

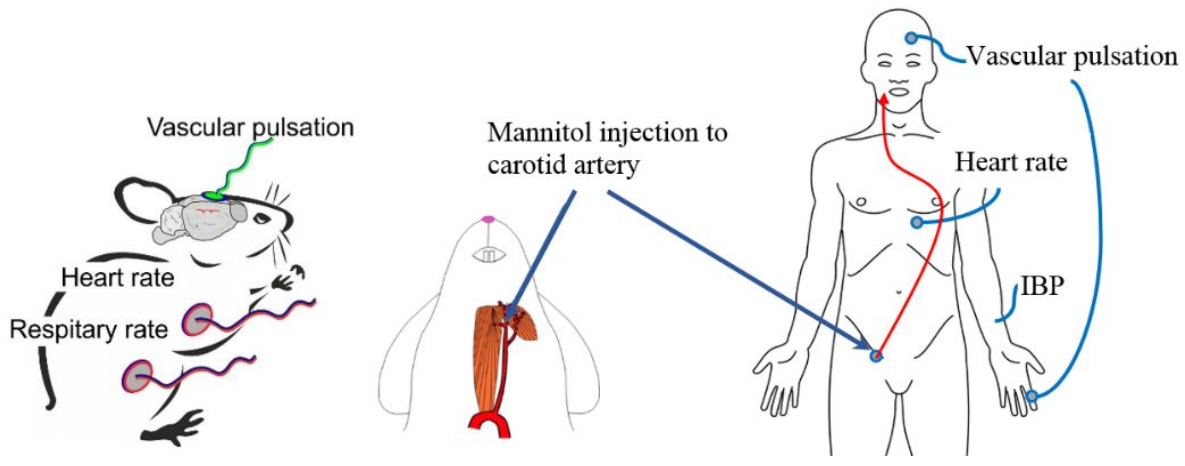

Figure 1. Placement of the sensors for measuring HR, respiratory rate and vascular pulsation. Signals from mouse are measured continuously using same sensing techniques that we utilise for human measurements [10 - 12]. In the middle, mannitol is injected via a catheter from external carotid into internal carotid artery under microscopy guidance. In right, human mannitol is injected to a catheter traversing via femoral artery and aorta into the internal carotid artery (red line). The catheter is placed in x-ray guided fluoroscopy [6]. In addition, BP was measured on human patients invasively (IBP). 


\subsection{BBBD by intra-arterial mannitol infusion}

$B B B D$ procedure in mouse: 2-months-old mice of C57/B16 background were anesthetized with isoflurane (2\%). External carotid artery was exposed and thin $(<200 \mu \mathrm{m})$ heparine filled cannula was introduced into the vessel. Prewarmed $25 \%$ mannitol or $0,9 \% \mathrm{NaCl}$ (saline) supplemented with $2 \%(\mathrm{~W} / \mathrm{V})$ Evans blue dye (EB) was infused into circulation in $240 \mu \mathrm{l}$ volume. The infusion rate was set to attain infusion duration of $30 \mathrm{sec}$. The infusion solutions were let to circulate for $20 \mathrm{~min}$ after which the animal was euthanized with overdose of Ketamine-Xylazine. The remaining EB in the vasculature was drained via contralater common carotid artery by introducing saline at the rate $500 \mu \mathrm{l} / \mathrm{min}$ for 10 min. EB was extracted from both hemispheres with formamide and absorbance measured with $620 \mathrm{~nm}$, in order to evaluate efficiency of BBBD.

Animal experiments were performed under the licence of National Animal Experiment Board of Finland (License number: Esavi/2362/04.10.07/2017 and Esavi/826/04.10.07/2018).

$B B B D$ procedure in human: BBBD procedure in Oulu University Hospital is based on the original procedure of Edward Neuwelt and coworkers [13,14]. Before anaesthesia induction intravenous phenobarbital and midazolam are given. Anaesthesia is induced and maintained using propofol. Two to three minutes prior to intra-arterial mannitol infusion anaesthesia is deepened up to EEG suppression level (entropy 0) with a $250 \mathrm{mg}$ intravenous thiopental bolus together with benzodiazepine. Atropine is given to counteract strong vasovagal effects of BBBD. Mannitol is given to one of the internal carotid arteries or to the dominant vertebral artery [14]. After angiographic verification of the selected artery, a hyperosmolal 25\% mannitol (Hospira, Inc., Lake Forest, IL) bolus is administered intra-arterially in $30 \mathrm{~s}$ at an infusion rate of 4-6 ml/s, followed by 10-minute intra-arterial infusions of first methotrexate and then carboplatin (infusion rate $0.2-0.4 \mathrm{ml} / \mathrm{min}$ ). Etoposide and cyclophosphamide are given intravenously $5-10 \mathrm{~min}$ prior to mannitol $[3,6]$.

BBBD procedures in human patients were carried out in Oulu University Hospital. Each patient was informed about data collection and signed the written consent prior to the procedure. The study was carried out in accordance with the Declaration of Helsinki and approved by the Ethical Committee of Northern Ostrobothnia Hospital District, Oulu University Hospital (number 5/2014).

\section{RESULTS}

Figure 2 shows typical cardiovascular effects monitored during the human BBBD by intra-arterial mannitol infusion $[3,6]$.

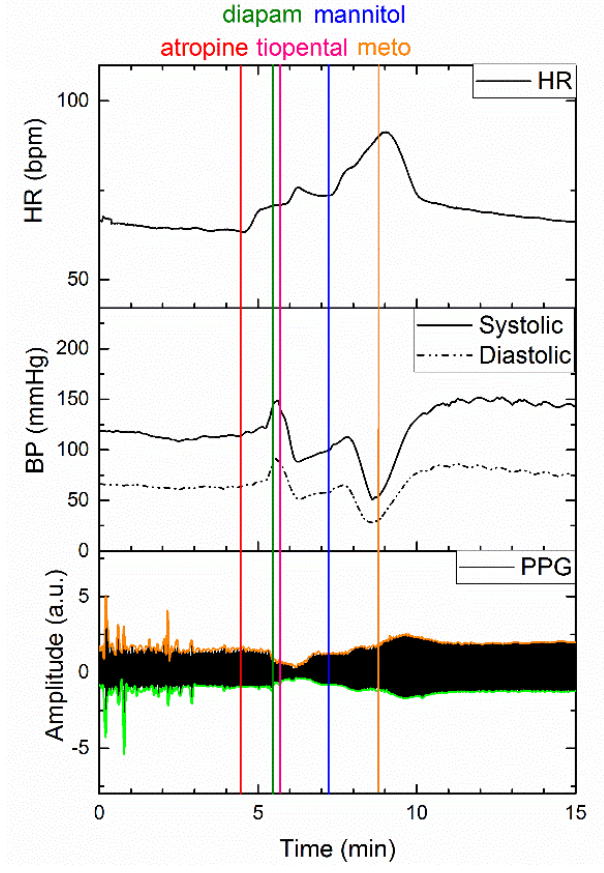

Figure 2. Cardiovascular effects in the BBBD procedure in human patients, caused by atropine, diapam, thiopental, mannitol and meto. 
First i.v. atropine increases HR after $30 \mathrm{~s}$ re-distribution delay. Thiopental i.v. injection decreases BP markedly. Then intra-carotid mannitol (blue vertical line) first increases BP and then causes subsequent drop of BP to $50 \mathrm{mmHg}$. This is countered by HR increase. After methotrexate injection into carotid artery increases the BP to the level of $150 \mathrm{mmHg}$ with simultaneous HR drop. Interestingly the BP shows vasomotor waves 4-5 min after mannitol injection.

Figure 3 shows typical cardiovascular effects of mannitol in mouse. Mannitol injection induces identical initial increase in HR followed by a drop. Respiration increases markedly during the HR drop. Furthermore, upper and lower envelopes of the PPG signal, reflecting vascular pulsation, correlate with the dynamics of HR, in both human and mouse responses.
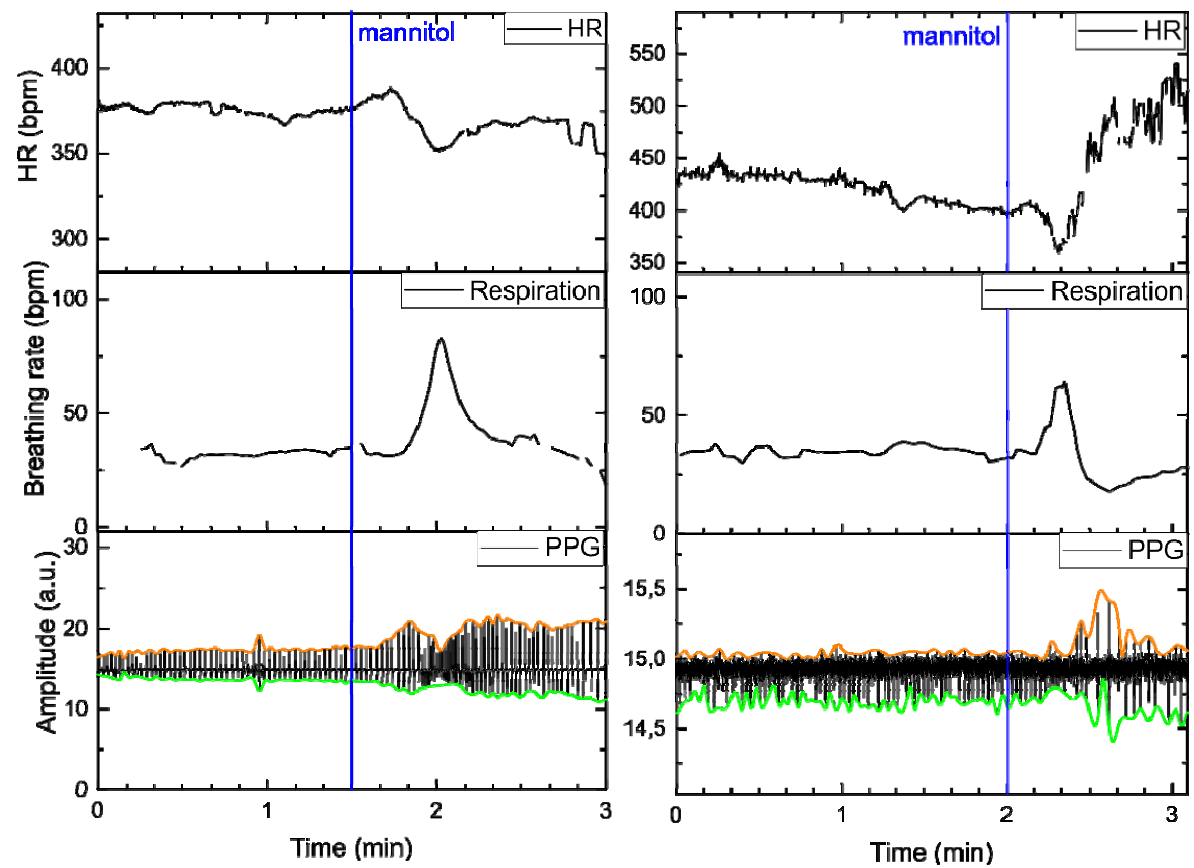

Figure 3. Typical cardiovascular effects of mannitol infusion in mouse including heart rate, respiration and vascular pulsation. $25 \%$ mannitol bolus in a volume of $240 \mu \mathrm{l}$ was injected into internal carotid artery for $30 \mathrm{~s}$ at the rate of 480 $\mu \mathrm{l} / \mathrm{min}$.
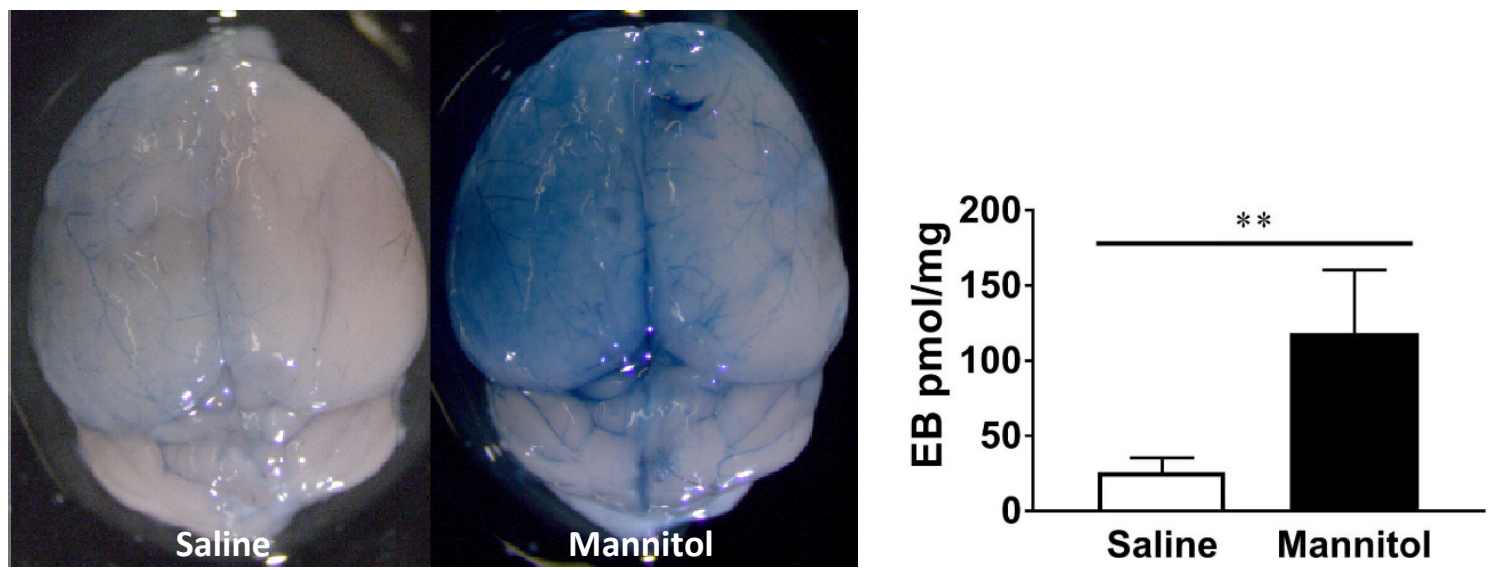

Figure 4. In order to evaluate efficiency of BBBD, $25 \%$ mannitol solution or physiological saline $(0,9 \% \mathrm{Nacl})$ was supplemented with 2\% (W/V) Evans blue (EB) and adminsitred into brain circulation via left extrernal carotid artery. Dye leakage and distribution in the parenchyma was evident especially on the side of mannitol infusion. Quantification of EB from left and right hemispheres (infusion bolus size $240 \mu 1$ and rate $480 \mu 1 / \mathrm{min}$ ). 


\section{DISCUSSION}

Here we show that similar opto-electro-mechanical sensors that are used in humans can also be applied to mice. However, due to small body size optimal positioning of the sensors and shaving of skin areas was crusial for detecting low amplitude oscillations. With proper filtering and by utilizing several sensors, placed on chest, abdomen and head, we were able to reduce motion artefacts and dissect oscillations emerging from respiration, heartbeats and blood pulsations.

Typically, mannitol injection induced identical response to human data (as presented in Figures 2 and 3). However, mouse vital functions showed noticeable variation between individuals. We think this is due to variable effect of isoflurane anesthesia to vascular tone. Isoflurane slows down the respiration rate [15], causes hypercapnia and cerebral vasodilation [16,17]. We see individual variation in baseline respiration rate this being generally lower than 50 cycles/min. Interestingly, some individuals with low baseline respiration rate developed tachycardia following mannitol bolus. Due to its variable effects on vascular tone and dampening influence on BBBD isoflurane is not preferred anesthetic in rats and humans [16]. However, we were able to induce significant BBBD reproducibly by using isoflurane and prefer its use due to its fast and effective anesthesia and easiness of post-anesthetic recovery.

Altogether, our mouse sensors can be used to monitor vital functions in mice during anesthezia and to assess cardiovascular and pulmonary responses when challenged.

\section{ACKNOWLEDGEMENTS}

This work was supported by Academy of Finland TERVA grant, JAES-Foundation and Novo Nordisk Foundation (TM).

\section{REFERENCES}

[1] Neuwelt, E. A., Frenkel, E. P., Diehl, J. T., Maravilla, K. R., Vu, L. H., Clark, W. K., Rapoport, S. I., Barnett, P. A., Hill, S. A., Lewis, S. E., Ehle, A. L., Beyer, C. W.,Jr and Moore, R. J. , "Osmotic blood-brain barrier disruption: a new means of increasing chemotherapeutic agent delivery," Trans.Am.Neurol.Assoc. 104, 256-260 (1979).

[2] Harjamaa, L., Kuitunen, H., Turpeenniemi-Hujanen, T., Haapasaari, K. M., Leppä, S., Mannisto, S., Karjalainen-Lindsberg, M., Lehtinen, T., Eray, M. and Vornanen, M. , "Constant pattern of relapse in primary central nervous lymphoma patients treated with high-dose methotrexate combinations. A Finnish retrospective study," Acta Oncol. 54(6), 939-943 (2015).

[3] Kuitunen, H., Tokola, S., Siniluoto, T., Isokangas, M., Sonkajärvi, E., Alahuhta, S., Turpeenniemi-Hujanen, T., Jantunen, E., Nousiainen, T. and Vasala, K. , "Promising treatment results with blood brain barrier disruption (BBBD) based immunochemotherapy combined with autologous stem cell transplantation (ASCT) in patients with primary central nervous system lymphoma (PCNSL)," J.Neurooncol. 131(2), 293-300 (2017).

[4] Burgess, A., Ayala-Grosso, C. A., Ganguly, M., Jordão, J. F., Aubert, I. and Hynynen, K. , "Targeted delivery of neural stem cells to the brain using MRI-guided focused ultrasound to disrupt the blood-brain barrier," PloS one 6(11), e27877 (2011).

[5] Semyachkina-Glushkovskaya, O., Kurths, J., Borisova, E., Sokolovski, S., Mantareva, V., Angelov, I., Shirokov, A., Navolokin, N., Shushunova, N. and Khorovodov, A. , "Photodynamic opening of blood-brain barrier," Biomedical optics express 8(11), 5040-5048 (2017).

[6] Kiviniemi, V., Korhonen, V., Kortelainen, J., Rytky, S., Keinänen, T., Tuovinen, T., Isokangas, M., Sonkajärvi, E., Siniluoto, T., Nikkinen, J., Alahuhta, S., Tervonen, O., Turpeenniemi-Hujanen, T., Myllylä, T., Kuittinen, O. and Voipio, J., "Real-time monitoring of human blood-brain barrier disruption," PloS one 12(3), e0174072 (2017).

[7] Garcia-Sola, R., Pulido, P. and Capilla, P. , "The immediate and long-term effects of mannitol and glycerol," Acta Neurochir. 109(3-4), 114-121 (1991).

[8] Gumerlock, M. K. and Neuwelt, E. A. , "The effects of anesthesia on osmotic blood-brain barrier disruption," Neurosurgery 26(2), 268-277 (1990).

[9] Van Gastel, M., Stuijk, S. and De Haan, G. , "New principle for measuring arterial blood oxygenation, enabling motion-robust remote monitoring," Scientific reports 6, 38609 (2016). 
[10] Myllylä, T., Zacharias, N., Korhonen, V., Zienkiewicz, A., Hinrichs, H., Kiviniemi, V. and Walter, M. , "Multimodal brain imaging with magnetoencephalography: A method for measuring BP and cardiorespiratory oscillations," Scientific reports 7(1), 172 (2017)

[11] Myllylä, T., Korhonen, V., Suraźyński, Ł., Zienkiewicz, A., Sorvoja, H. and Myllylä, R. , "Measurement of cerebral blood flow and metabolism using high power light-emitting diodes," Measurement 58, 387-393 (2014).

[12] Myllylä, T., Korhonen, V., Vihriälä, E., Sorvoja, H., Hiltunen, T., Tervonen, O. and Kiviniemi, V. , "Human heart pulse wave responses measured simultaneously at several sensor placements by two MR-compatible fibre optic methods," Journal of Sensors, 2012 (2012).

[13] Angelov, L., Doolittle, N. D., Kraemer, D. F., Siegal, T., Barnett, G. H., Peereboom, D. M., Stevens, G., McGregor, J., Jahnke, K., Lacy, C. A., Hedrick, N. A., Shalom, E., Ference, S., Bell, S., Sorenson, L., Tyson, R. M., Haluska, M. and Neuwelt, E. A. , "Blood-brain barrier disruption and intra-arterial methotrexate-based therapy for newly diagnosed primary CNS lymphoma: a multi-institutional experience," J.Clin.Oncol. 27(21), 3503-3509 (2009).

[14] Neuwelt, E. A., Maravilla, K. R., Frenkel, E. P., Rapaport, S. I., Hill, S. A. and Barnett, P. A. , "Osmotic bloodbrain barrier disruption. Computerized tomographic monitoring of chemotherapeutic agent delivery," J.Clin.Invest. 64(2), 684-688 (1979).

[15] Tsukamoto, A., Serizawa, K., Sato, R., Yamazaki, J. and Inomata, T. , "Vital signs monitoring during injectable and inhalant anesthesia in mice," Experimental animals 64(1), 57-64 (2015).

[16] Remsen, L. G., Pagel, M. A., McCormick, C. I., Fiamengo, S. A., Sexton, G. and Neuwelt, E. A. , "The influence of anesthetic choice, $\mathrm{PaCO} 2$, and other factors on osmotic blood-brain barrier disruption in rats with brain tumor xenografts," Anesthesia \& Analgesia 88(3), 559-567 (1999).

[17]Eger, E. I. , "IsofluraneA Review," Anesthesiology: The Journal of the American Society of Anesthesiologists 55(5), 559-576 (1981). 